\title{
O MAUP e a Análise Espacial: um Estudo de Caso para o Rio Grande do Sul (1991-2000)'
}

\author{
Rodrigo Peres de Ávila* \\ Leonardo Monasterio*
}

Resumo: O trabalho apresenta um caso de MAUP (Modifiable Areal Unit Problem) com dados de renda per capita para o Rio Grande do Sul no período 1991-2000. É demonstrada a necessidade de se considerarem as diferenças nos resultados obtidos oriundas apenas da regionalização escolhida pelo pesquisador. A análise se centra em dois níveis: municípios e Conselhos Regionais de Desenvolvimento. Aplica-se a Análise Exploratória de Dados Espaciais (ESDA) para identificar suas associações espaciais globais e seus clusters. Em seguida, fazemse os tradicionais testes de $\beta$-convergência condicional e absoluta, acrescentando a econometria espacial e comparando os resultados com o modelo de econometria clássico. A análise de kernel estocástico nos dois níveis de análise complementa o estudo. Mostra-se que os resultados dependem do nível espacial e que há processos que só são identificados após avaliação cuidadosa dos dados. A conclusão principal é de que o MAUP é um fenômeno relevante, que não pode deixar de ser considerado pelos economistas regionais.

Palavras-chave: Econometria espacial, Kernel Estocástico, MAUP - ESDA.

Abstract: The paper presents a case of MAUP (Modifiable Areal Unit Problem)
using income per capita data of Rio Grande do Sul (1991-2000). It discusses and
shows the need of considering the different results that emerge from different
choices of data aggregation. There are two levels of analysis: municipalities and
Regional Development Councils (COREDEs). Exploratory Spatial Data Analysis
(ESDA) identifies global spatial correlations and its clusters. The tests of $\beta$-con-
vergence are done, using classic and spatial econometrics. The study is comple-
mented by stochastic kernel analyses on COREDEs and municipalities. It is shown
that results change according to the spatial level, and there are processes that
only emerge after a careful assessment of data. The paper concludes that MAUP
is a relevant issue and that should not be overlooked by regional economists.

Keywords: Spatial Econometrics, Stochastic Kernel, MAUP, ESDA.

Jel Codes: R11.

1 Agradecemos a revisão de Martin Dietrich Brauch. Os erros são de responsabilidade dos autores.

* Doutorando em Economia Aplicada na Universidade Federal do Rio Grande do Sul UFRGS

** Professor do Departamento de Economia da Universidade Federal de Pelotas - UFPel

Recebido em 20 de novembro de 2006. Aceito em 18 de junho de 2008. 


\section{Introdução}

A análise espacial de dados vem sendo lentamente incorporada pelos economistas regionais. Enquanto esse ramo de pesquisa avança no front teórico, há todo um conjunto de ferramentas subexploradas pelos analistas. Nesse sentido, o objetivo deste trabalho é aplicar o instrumental da análise e da econometria espacial para a questão da distribuição da renda per capita no território gaúcho entre 1991 e 2000.

Mais ainda, pretende-se ilustrar o problema da unidade de área modificável. O chamado MAUP (Modifiable Areal Unit Problem MAUP, doravante) ${ }^{2}$ é bastante conhecido pelos geógrafos, mas pouco estudado pelos economistas. Não obstante, estes o enfrentam de qualquer modo. Trata-se, grosso modo, dos problemas decorrentes das distintas formas de delimitar as unidades espaciais. Diferentes desenhos das unidades implicam em diferentes análises, inferências ou mesmo políticas. A situação de um município pobre em uma área rica deixa de ser corretamente avaliada quando o nível de agregação espacial é a região, e a própria delimitação dos recortes regionais altera os resultados dos indicadores estatísticos.

O presente trabalho ilustra um caso de MAUP a partir de uma base de dados conhecida: os dados de renda per capita oriundos dos Censos Demográficos de 1991 e 2000. A análise se centra em dois níveis: municípios e Conselhos Regionais de Desenvolvimento (COREDEs). Aplica-se a Análise Exploratória de Dados Espaciais (ESDA, em inglês) a essas bases para identificar suas associações espaciais globais e seus clusters. Em seguida, fazem-se os tradicionais testes de $\beta$ convergência condicional e absoluta, acrescentando a econometria espacial. Em todos os resultados das análises fica claro que houve interferência do MAUP. Vale apontar que os trabalhos de convergência com econometria espacial que utilizaram dados brasileiros ignoraram tal problema. Magalhães, Hewings e Azzoni (2000), Mossi et al. (2002) e Magalhães (2001), Pimentel e Haddad (2004), Verner e Tebaldi (2004), e Monasterio e Avila (2005) trataram apenas de um nível espacial. Esses autores desconsideraram o risco que seus resultados fossem específicos ao grau de agregação escolhido.

Este trabalho enfatiza a necessidade de maior atenção aos dados ao se fazerem testes de convergência. Aparentes processos de convergência podem ocorrer, como será aqui mostrado, por meca-

\footnotetext{
2 Optou-se por manter a sigla em Inglês não só porque ela já está consagrada, mas também pelo fato de que a sigla traduzida não seria eufônica.
} 
nismos bem diferentes dos previstos pelos modelos teóricos. Por fim, há que se ressaltar que a abordagem do trabalho é empírica. Os testes aqui apresentados são adequados muito mais para identificar padrões e tendências espaciais do que para cotejar teorias. Outros autores já se voltaram para o estudo da desigualdade e da convergência de renda no Rio Grande do Sul com maior atenção às questões teóricas. Nesta ampla literatura, destaca-se o trabalho fundamental de Alonso (1986), além de Bandeira (1994), Porto Jr. (2000), Porto Jr. e Ribeiro (2000), Berni et al. (2002), Bagolin et al. (2002), Marqueti e Ribeiro (2002), Alonso e Amaral (2004) e Souza (2004).

\section{0 problema da unidade de área modificável (MAUP)}

"Uma mesma estatística calculada sobre duas unidades espaciais costuma gerar diferenças de acordo com o recorte utilizado, mesmo que os dados originais sejam os mesmos. Essa é a natureza do MAUP" (HAINING, 2003). Na verdade, ele surge da própria necessidade de se agregarem informações espaciais. Essa agregação pode ser necessária porque não se têm dados individuais, ou porque o próprio objeto de análise são unidades agregadas. Por exemplo, suponha que há uma correlação entre dados eleitorais de um partido político e dados de violência por zona eleitoral. Isto não permite afirmar que seus eleitores são mais propensos à violência. Pode ser que os violentos sejam outros indivíduos e a correlação estatística seja uma ilusão. Inferir de forma equivocada o comportamento individual com base em dados agregados constitui a chamada falácia ecológica. ${ }^{3}$ Uma ilustração, no caso da Economia, é quando se considera que diminuiu o número de pessoas pobres em uma região, porque a sua renda per capita média aumentou.

Haining (2003, p. 150) aponta dois tipos de MAUP nas propriedades estatísticas: um decorre dos diferentes níveis possíveis de agregação das unidades espaciais; o outro decorre do próprio recorte das unidades (mantido o nível constante). O primeiro é chamado de problema de escala e o outro é chamado de o problema de partição.

A figura abaixo apresenta o problema de partição. Suponha que cada ponto significa, por exemplo, uma empresa de um certo ramo, enquanto as linhas são as fronteiras das unidades de análise. No caso II, o pesquisador que olhasse apenas os dados da distribuição de tal atividade - e não o mapa - consideraria que ela estava

\footnotetext{
3 De certa forma, a falácia ecológica é o erro oposto à falácia da composição.
} 
bem distribuída pelas quatro unidades. Já se as fronteiras das unidades fossem como as que estão descritas em I, apenas um pouco diferentes de II, o pesquisador identificaria que tal ramo estava totalmente concentrado (QUAH e SIMPSON, 2003).

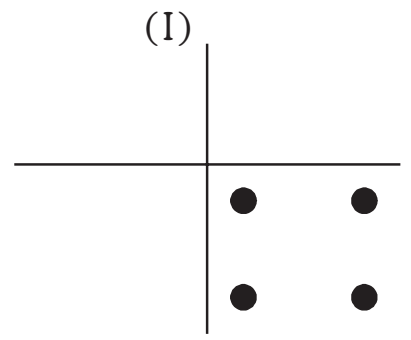

Figura 1. MAUP: o problema de partição

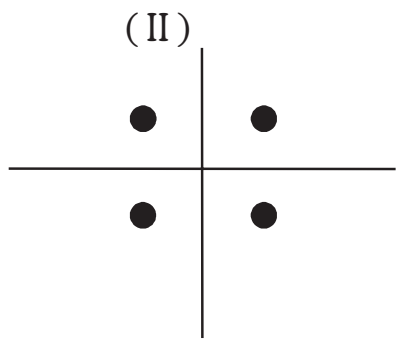

Fonte: QUAH e SIMPSON (2003, p. 10).

Sobre o problema de escala, tome-se a ilustração abaixo. Levando-se em conta o nível mais alto de agregação, isto é, os quadrados e não os retângulos que os compõem, os dados mostrariam que, entre as duas datas, a área $\mathrm{A}$ passou de 3 para 4 unidades, enquanto a B passou de 2 para também 4 . Ou seja, o retrato é de aumento da semelhança entre as duas áreas.

Porém, considerando-se os retângulos, a imagem é distinta. Na área A, aquela sub-área que possuía uma unidade seguiu com o mesmo número. E na região $\mathrm{B}$, uma das que possuía uma unidade ficou vazia, enquanto a outra, saltou para quatro unidades. Ou seja, a evolução das sub-áreas mostra uma dinâmica bem distinta: elas parecem cada vez mais dessemelhantes entre si. Quem tivesse observado apenas o grau mais alto de agregação, teria ignorado a dinâmica interna das unidades.

1991

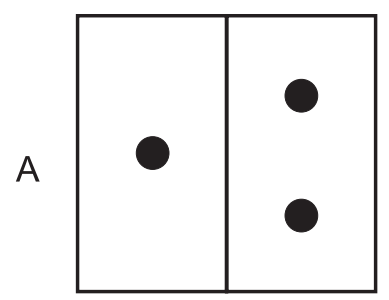

2000

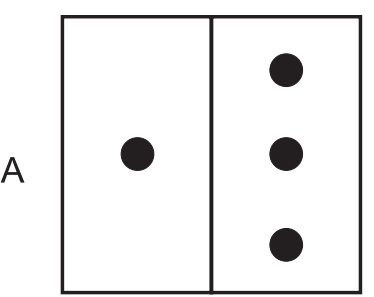



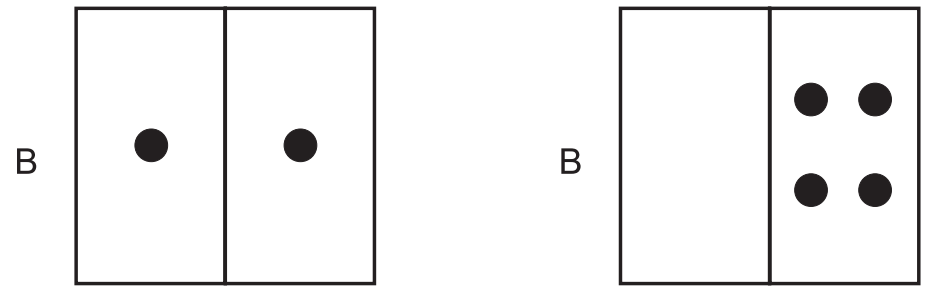

Figura 2. MAUP: o problema da escala

Fonte: elaboração dos autores

\section{Análise espacial da Renda per capitano Rio Grande do Sul (1991-2000)}

\subsection{MORAN-I}

O primeiro passo da análise consiste em buscar representações e testes que revelem se os dados estão relacionados espacialmente. Para tal, o gráfico e a estatística I de Moran são os instrumentos básicos. Esta é calculada da seguinte forma (REY e MONTOURI, 1999):

Onde:

$$
I=\frac{\sum_{i=1}^{n} \sum_{j=1}^{n} w_{i j} x_{i} x_{j}}{\sum_{j=1}^{n} x_{i}^{2}}
$$

$\mathrm{n}=$ número de observações

$w_{\mathrm{ij}}=$ elementos da matriz de contigüidade binária normalizada (W). Se os elementos, no caso as unidades espaciais, i e j forem contíguos, $\mathrm{w}_{\mathrm{ij}}$ terá valor 1 ; caso contrário, 0 . A matriz deve ser normalizada pelo total das linhas.

$\mathrm{x}_{\mathrm{i}}$ e $\mathrm{x}_{\mathrm{i}}=$ valores da variável analisada em desvios da média.

Quando a estatística I de Moran é próxima de +1 significa que existe uma autocorrelação positiva, ou seja, valores altos (baixos) tendem a estar localizados na vizinhança de valores altos (baixos). Se for próximo de -1, o inverso ocorre: valores altos estarão cercados de valores baixos, e vice-versa. ${ }^{4}$ Conforme imaginado, quando ele é zero, não há autocorrelação espacial.

\footnotetext{
4 Um tabuleiro de xadrez é a melhor representação da autocorrelação inversa perfeita (I de Moran = -1). Vale apontar que, neste trabalho, o critério de contigüidade adotado foi o chamado Queen. Ou seja, foram consideradas vizinhas as unidades que compartilhassem fronteiras ou vértices com outras.
} 
O gráfico de Moran (Moran scatterplot) é uma representação visual da estatística I. Ele apresenta o valor padronizado de uma variável para cada uma das unidades (no caso, a renda per capita) nas abscissas e, no eixo das ordenadas, a média do valor padronizado da mesma variável para os vizinhos destas unidades. Desta maneira, valores acima da média com vizinhança também acima da média ocuparão o primeiro quadrante. Já aqueles abaixo da média, com vizinhos na mesma situação ocupam o terceiro quadrante. Os segundos e quartos quadrantes são ocupados, respectivamente, por unidades que sejam ilhas elevadas cercadas por valores baixos, e por bolsões baixos cercados de valores altos. Caso não houvesse qualquer correlação espacial, a nuvem de pontos estaria bem distribuída pelos quatro quadrantes.

\subsubsection{Moran-I dos Municípios}

De início, apresenta-se a representação gráfica da estatística de Moran $^{5}$ verificada para o Rio Grande do Sul. Os gráficos referem-se à renda per capita de 1991, ao crescimento da renda per capita no período 1991-2000 e à renda per capita de 2000, respectivamente.

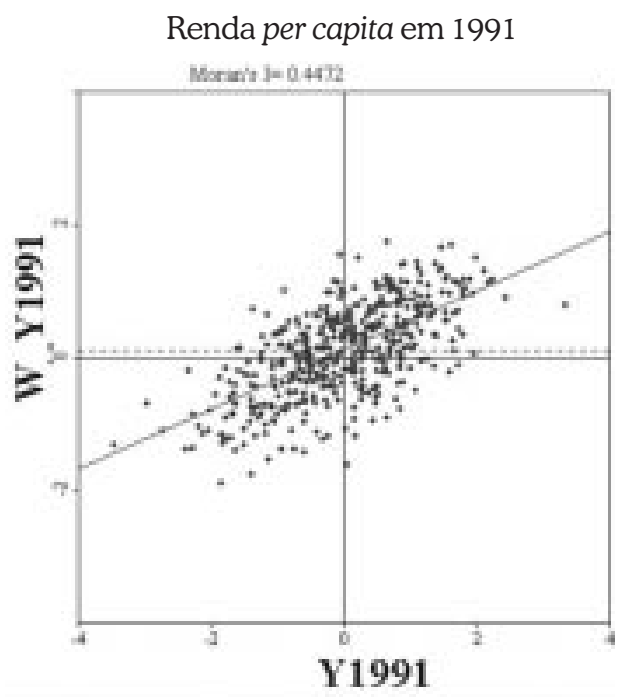

5 Os testes espaciais foram realizados através do software Geoda095i-Beta. O programa está disponível no sítio http:// www.geoda.uiuc.edu. Vale notar que não foi feito ajuste para os efeitos de fronteira. Essa é uma das limitações dos métodos de análise espacial. 
Taxa de crescimento da Renda per capita (1991-200)

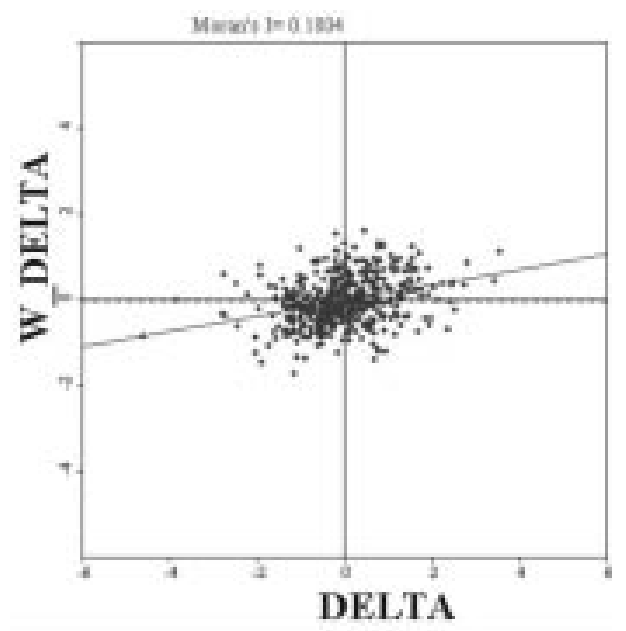

Renda per capita em 2000

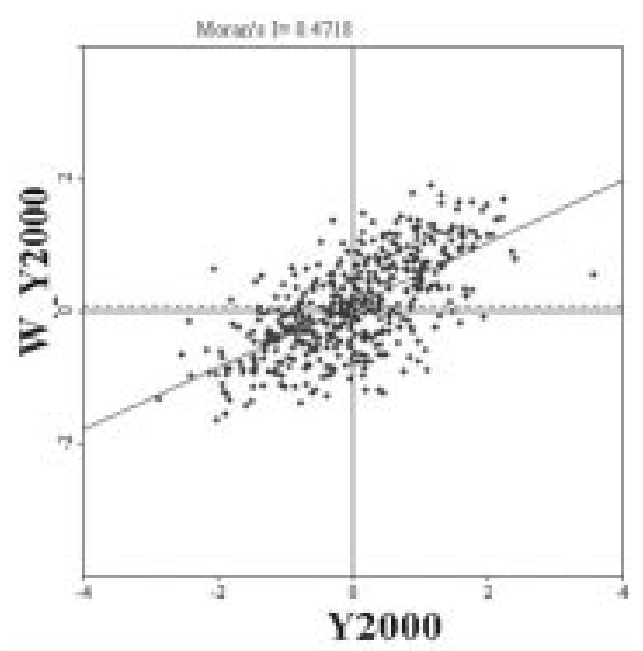

Figura 3. Gráfico I de Moran para a Renda per capita municipal (1991-2000) Fonte: cálculos dos autores

Os três testes mostraram-se estatisticamente significativos. Os pontos representam os municípios gaúchos. Nota-se que há maior concentração de pontos nos quadrantes que representam valores semelhantes entre a unidade analisada e suas vizinhas (primeiro e terceiro quadrantes). O I de Moran relativo ao ano de 1991 apresenta valor igual a 0,4472. A visualização da estatística nos mostra 
que no Rio Grande do Sul, em 1991, houve tendência de que municípios mais ricos que a média estivessem espacialmente associados com municípios igualmente mais ricos que a média. Da mesma forma, municípios mais pobres que a média estiveram contíguos a municípios igualmente mais pobres.

Nota-se que, embora com menor intensidade, em relação ao crescimento da renda per capita também há maior concentração de pontos nos quadrantes que representam valores semelhantes entre a unidade analisada e suas vizinhas (primeiro e terceiro quadrantes). O I de Moran relativo ao crescimento da renda no período 1991-2000 apresenta valor igual a 0.1804 . A visualização do gráfico nos permite dizer que houve tendência de concentração espacial de municípios que apresentaram ritmos de crescimento da renda per capita semelhantes.

Por fim, de maneira análoga a 1991, nota-se que no ano 2000 há maior concentração de pontos nos quadrantes que representam valores semelhantes entre a unidade analisada e suas vizinhas (primeiro e terceiro quadrantes). O I de Moran relativo ao ano de 2000 apresenta valor igual a 0,4718. A visualização da estatística nos mostra que no Rio Grande do Sul, em 2000, também houve tendência de que municípios mais ricos que a média estivessem espacialmente associados com municípios igualmente mais ricos que a média. Da mesma forma, municípios mais pobres que a média estiveram espacialmente associados com municípios igualmente mais pobres que a média.

\subsubsection{Moran-I dos COREDEs}

O valor do teste de Moran-I para a renda per capita em 1991 por COREDE, 0,1658, foi estatisticamente significativo a 5\%. Na figura 4 os pontos representam os COREDEs no Rio Grande do Sul e há uma moderada concentração nos quadrantes que representam valores semelhantes entre a unidade analisada e suas vizinhas (primeiro e terceiro quadrante). Assim, a visualização da estatística nos mostra que houve uma leve tendência de que COREDEs mais ricos que a média estivessem espacialmente associados aos COREDEs igualmente mais ricos. Em relação à taxa de crescimento da renda per capita entre 1991-2000, o mesmo fenômeno se deu. O I de Moran apresenta valor igual a 0.3590 , indicado que o desempenho dos COREDEs esteve espacialmente associado de forma intensa.

Já para 2000, as rendas per capita estão espacialmente correlacionadas. O valor baixo do Moran-I, 0,1151, só o torna estatisticamente diferente de zero se forem adotados graus generosos de 
significância. Sintetizando os resultados, a renda em 1991 e o crescimento entre 1991-2000 apresentam correlação espacial, mas o mesmo não acontece com a renda em 2000. Esse contraste não é tão estranho quanto parece à primeira vista. Houve um cluster de COREDEs localizados no norte do estado que teve uma taxa de crescimento mais alta que a média (fazendo com que ficassem todos no primeiro quadrante do gráfico). Isto fez com que, em relação em 2000, a distribuição espacial da renda per capita não estivesse tão concentrada quanto em 1991.

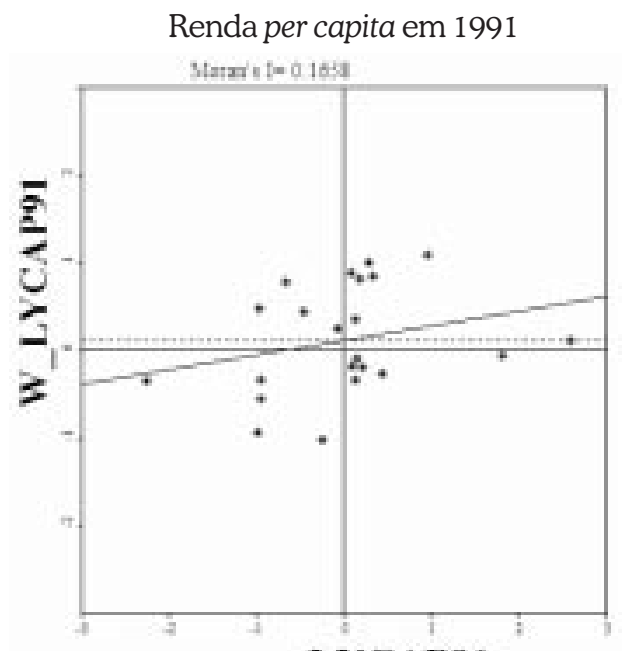

LYCAP91

Taxa de crescimento da Renda per capita (1991-200)

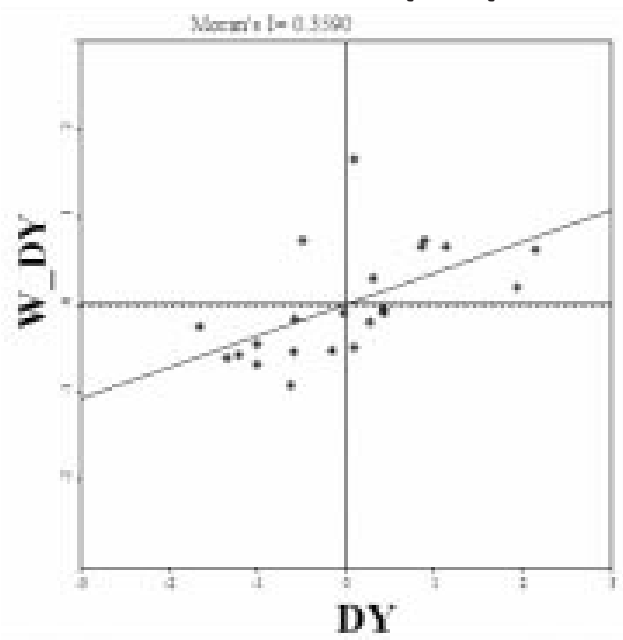

Ávila, R.P.; Monasterio, L. OMAUP ea Análise Espacial: um Estudo... 


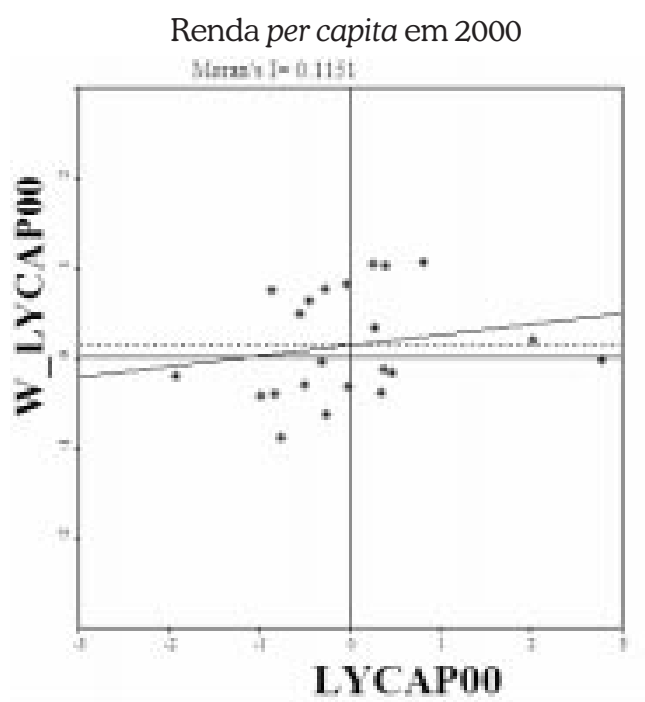

Figura 4. Gráfico I de Moran para a Renda per capita por COREDE (1991-2000) Fonte: cálculos dos autores

\subsection{Clusters Epaciais}

Anselin (1995) definiu que um Local Indicator of Spatial Association (LISA) deveria ter duas propriedades: a) apontar aquelas unidades em redor da qual há aglomeração de valores semelhantes; b) a soma dos LISA individuais deveria ser proporcional ao indicador de associação geral (como o Moran I). O indicador de Moran Local (Ii) guarda essas características e pode ser calculado da seguinte forma:

$$
I_{i}=\frac{x_{i} \sum_{j} w_{i j} x_{j}}{\sum_{i} x_{i}^{2}}
$$

Valores de $\mathrm{I}_{\mathrm{i}}$ estatisticamente diferentes de 0 indicam que a unidade i está espacialmente associada aos seus vizinhos. Como a distribuição dos $I_{i}$ é desconhecida, a forma de obtê-la é através de permutações aleatórias dos vizinhos de cada unidade. A comparação destas com a observada permite inferir se a correlação espacial é significativa, ou seja, se se trata efetivamente de um cluster espacial.

Analogamente ao Indicador global, valores próximos de +1 nos remetem à existência de relação espacial do tipo High-High e LowLow. Valores próximos de -1 nos remetem à existência de relação espacial do tipo High-Low e Low-High. Valores próximos de zero 
nos indicam que a unidade não está significativamente associada espacialmente aos seus vizinhos.

\subsubsection{Clusters Espaciais nos Municípios}

Conforme já exposto, o Indicador Local nos empresta uma visão específica da distribuição espacial das variáveis. Nos gráficos que se seguem, estão representados os grupos estatisticamente significativos.

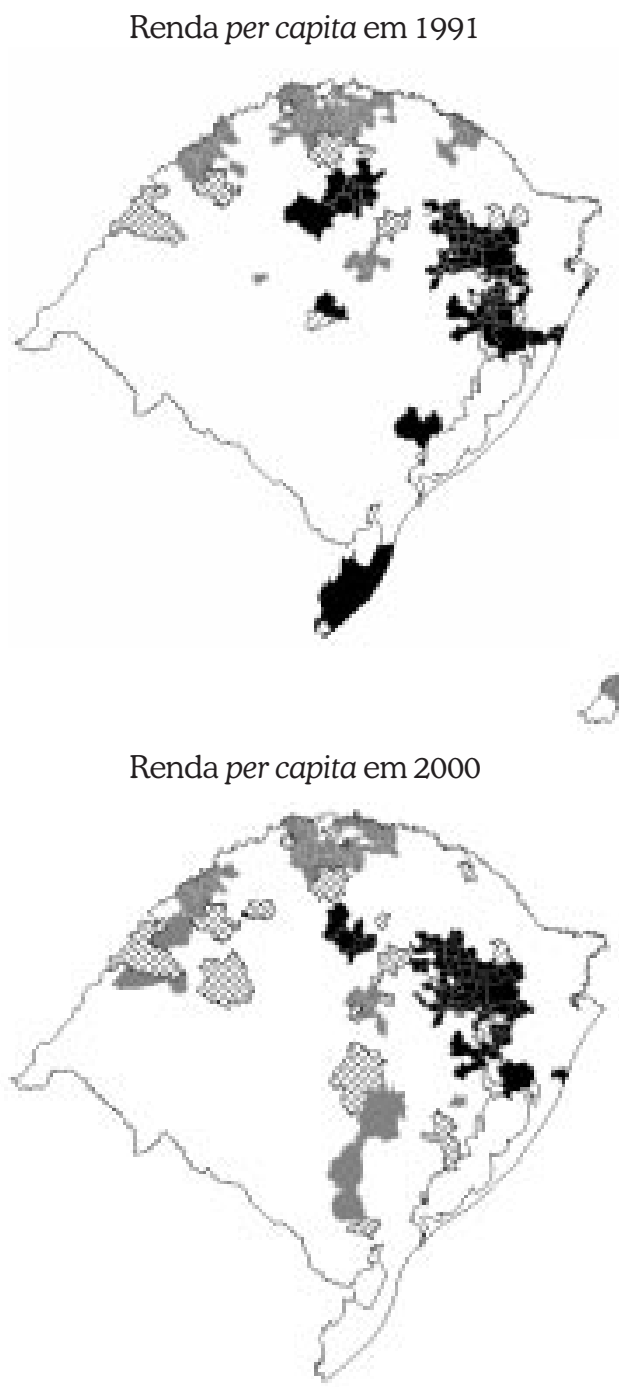

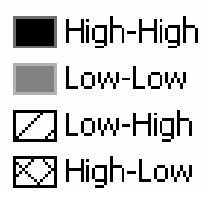

Taxa de crescimento da Renda per capita (1991-200)

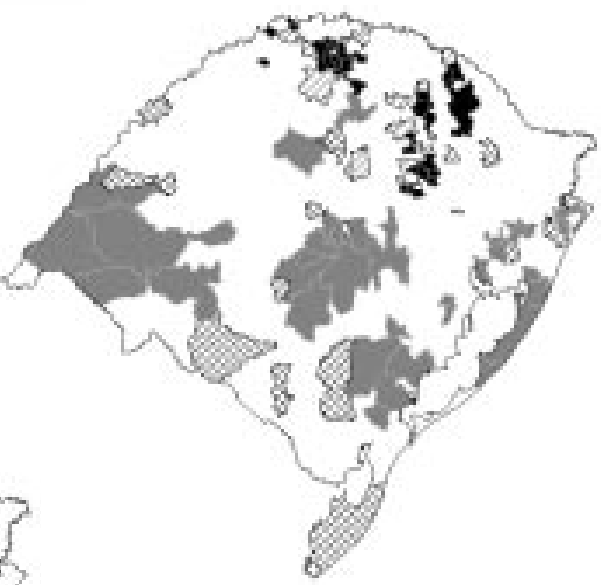

Figura 5. Cluster Map da Renda per capita por COREDE (1991-2000)

Fonte: cálculos dos autores 
Em 1991, nota-se um cluster de riqueza concentrado no core da economia gaúcha, o eixo que liga a região metropolitana de Porto Alegre até Caxias do Sul. Além disso, existe outro agrupamento que inclui Cruz Alta e municípios dos arredores. Os outros clusters são menores e mais esparsos pelo estado. O cluster Low-Low está localizado no extremo norte do Rio Grande do Sul. Os municípios mais representativos desse grupo são: Erval Seco, Planalto, Alpestre, Santo Cristo, Tenente Portela, Constantina, Redentora, Coronel Bicaco, Porto Xavier e Seberi. É interessante a constatação de que há alguns municípios com renda acima da média próximos a tais grupos pobres, o que nos remete ao padrão de distribuição espacial denominado High-Low.

Ao se observarem os agrupamentos de municípios que tiveram altas taxas de crescimento, os principais destaques - em termos populacionais - desse grupo são: Uruguaiana, Itaqui, Alegrete e Rosário do Sul. Ainda: Pelotas, Cachoeira do Sul, Canguçu, Rio Pardo e São Lourenço. E por fim: Novo Hamburgo, Gravataí e Taquara.

Há um grupo de alto crescimento econômico no norte gaúcho, justamente entre os municípios que tinham os menores níveis de renda em 1991. Tal constatação seria, a princípio, bem-vinda. Municípios como Três Palmeiras, Cerro Grande, Liberato Salzano Jaboticaba, Pinhal, Boa Vista das Missões, Seberi, Constantina, Cristal do Sul e São José das Missões fazem parte do cluster High-High, ou seja, tiveram altas taxas de crescimento da renda per capita. Contudo, todos estes municípios tiveram taxas negativas dos contingentes populacionais entre 1991 e 2000. A expulsão de sua população levou a um aumento da renda per capita, por migração seletiva. Isto é, foi a emigração dos mais pobres que elevou a renda per capita média destes municípios. Portanto, trata-se de um mecanismo de convergência que diferente daquele sugerido pelos modelos de crescimento tradicionais.

Em 2000, os agrupamentos de renda per capita tiveram basicamente o mesmo perfil de quase uma década antes. Mesmo nos municípios supracitados que tiveram aumento de renda per capita associada à perda populacional, a variação não foi suficiente para que deixasse de existir um cluster de pobreza municipal. É interessante apontar que, em 2000, na região Sul, desapareceram os esparsos municípios que possuíam o padrão High-High.

\subsubsection{Clusters Espaciais nos COREDEs}

A figura 6 deixa claro o problema de MAUP. Comparando os mapas, feitos a partir da mesma base de dados, os resultados dos 
clusters significativos de COREDEs são bastante distintos dos observados no mapa municipal. A aglomeração de municípios pobres no COREDE Médio-Alto Uruguai desaparece do mapa de significância. Por outro lado, até o litoral surge como um cluster significativo High-High. Em termos de crescimento da renda per capita, também há diferenças nos mapas: o COREDE Campanha se mostra como centro de um cluster Low-Low, sendo que seus municípios não estavam assim identificados no mapa municipal. Já no mapa de aglomerações significativas em sentido espacial e estatístico para a renda em 2000, restou apenas um cluster de renda alta formada pelos COREDEs Vale do Rio dos Sinos, Caí e Taquari.

Vale olhar mais de perto o COREDE Noroeste Colonial, que aparece como um dos membros da aglomeração de alto crescimento. De fato, sua renda per capita aumentou de $\mathrm{R} \$ 163$ para cerca de $R \$ 247$ entre 1991 e 2000. O peculiar é que o COREDE perdeu 7,6 mil habitantes no mesmo período. Portanto, tal como nos dados municipais, é preciso cuidado ao analisar os dados por COREDE. A migração seletiva em uma economia estagnada foi responsável, em parte, por este resultado, e não houve impulso dinâmico neste COREDE.

Renda per capita em 1991

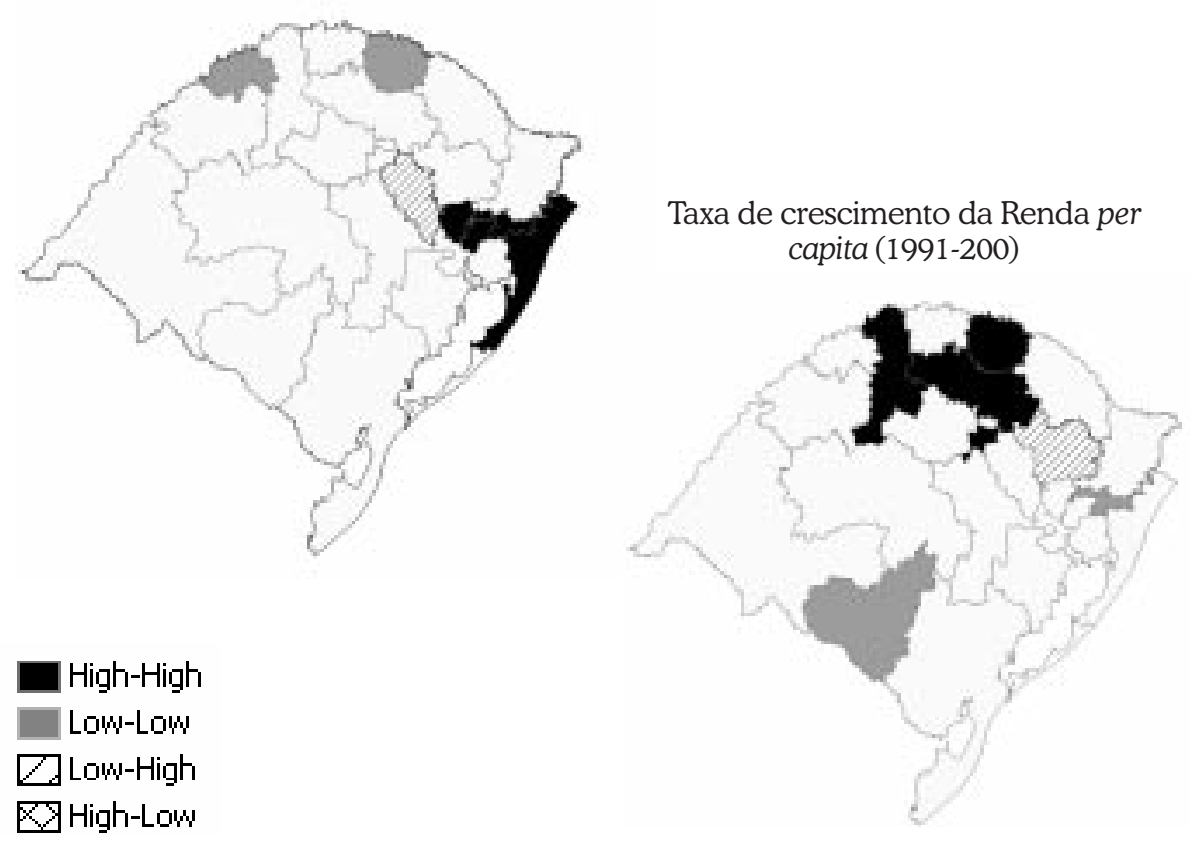




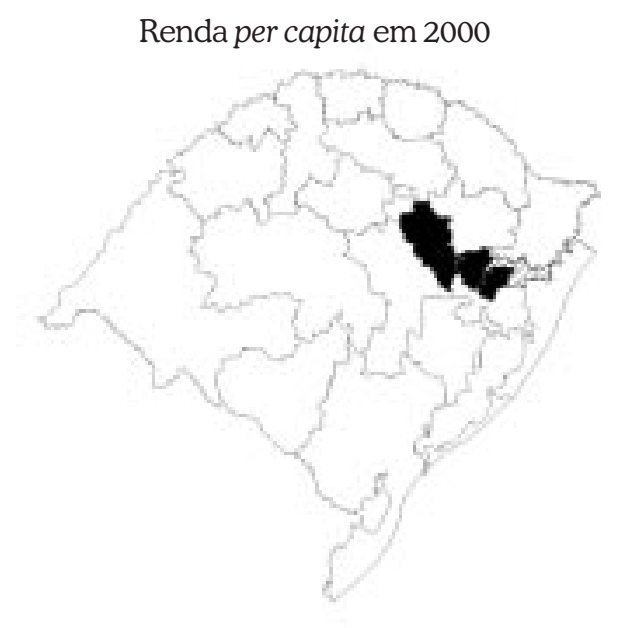

Figura 6. Cluster Map por COREDE da Renda per capita do Rio Grande do Sul (1991-2000)

Fonte: cálculos dos autores

\section{Convergência e Econometria Espacial no Rio Grande do Sul}

\subsection{Convergência e Análise Espacial}

Existe dependência espacial quando as variáveis dependentes ou erros em locais diferentes estão correlacionados entre si (ANSELIN, 1992). No caso presente, e na maior parte dos estudos sobre econometria espacial, imagina-se que a correlação espacial se dá entre áreas contíguas.

São duas as formas de autocorrelação espacial: a substantiva e a autocorrelação como "inconveniente", ou nuisance (ANSELIN, 1992, p. 176). No primeiro caso, os efeitos de transbordamento, ou externalidades espaciais de qualquer tipo, fazem com que as variáveis dependentes nas vizinhanças influenciem-se mutuamente. Por exemplo, em um estudo sobre os determinantes municipais da criminalidade, levar-se-iam em conta os níveis de crime nos municípios vizinhos. Havendo esse tipo de autocorrelação, a forma de corrigi-la se dá através da inclusão de chamados lags espaciais (em analogia com as defasagens temporais). Sua omissão levaria a modelos mal especificados, com estimadores viesados e inferências incorretas.

No outro tipo de autocorrelação espacial, são os erros que se acham espacialmente correlacionados. Isto pode decorrer de erros de medida. Como os limites das unidades geográficas muitas vezes não são os relevantes para as variáveis de interesse, os erros de unidades contíguas mostram-se dependentes (MAGALHÃES, 2001). As conseqüências de se omitir esse componente de autocorrelação 
espacial como nuisance são equivalentes à não correção de heteroscedasticidade: estimadores não-viesados, mas ineficientes, e, talvez, inferências estatísticas incorretas.

\subsection{Economia Espacial e as regressões de crescimento}

Como referência para os testes espaciais, parte-se do modelo mínimo de $\beta$-convergência proposto por Fuente (1996). Seguindo a notação e os procedimentos do autor (1996, p. 14), tem-se a seguinte forma funcional a ser testada:

Modelo I - Convergência Absoluta Clássica

$\Delta \mathrm{y}_{\mathrm{i}}=\mathrm{x}_{\mathrm{i}}+\beta \mathrm{y}_{\mathrm{i}, / 1991}+\varepsilon_{\mathrm{i}, \mathrm{t}}$

Onde:

$\mathrm{y}_{\mathrm{i}, \mathrm{t}}=$ In $\left(\mathrm{Q}_{\mathrm{it}} / \mathrm{Q}_{\mathrm{t}}\right)$, logaritmo da renda per capita da região i no período $t$ normalizada pela renda média estadual em t;

$\Delta \mathrm{y}_{\mathrm{i}}=\left(\mathrm{y}_{\mathrm{i}, 2000}-\mathrm{y}_{\mathrm{i}, 1991}\right) /(2000-1991)$

$\varepsilon_{\mathrm{i}, \mathrm{t}}=$ componente de erro;

$\mathrm{x}_{\mathrm{i}}=$ intercepto.

Chega-se ao modelo de dependência de erro espacial substituindo o componente de erro abaixo no Modelo I:

$\varepsilon_{\mathrm{t}}=\lambda \mathrm{W} \varepsilon_{\mathrm{t}}+\mathrm{u}_{\mathrm{t}}$

No qual $\lambda$ é um escalar do coeficiente do erro e $u_{t} \sim N\left(0, \sigma^{2} \mathrm{I}\right)$. O que resulta na forma funcional para o modelo de erro espacial:

Modelo II - Convergência Absoluta com Erro Espacial

$$
\Delta \mathrm{y}_{\mathrm{i}}=\mathrm{x}_{\mathrm{i}}+\beta \mathrm{y}_{\mathrm{i}, 1991}+(\mathrm{I}-\lambda \mathbf{W})^{-1} \varepsilon_{\mathrm{i}}
$$

A matriz $\mathbf{W}$ é a mesma matriz de contigüidade utilizada na estatística de Moran. Obviamente, quando o valor de l é igual a 0, não existe autocorrelação espacial do erro. Conforme chamaram a atenção Rey e Montouri (1999), quando $1^{1}$ 0, um choque ocorrido em uma unidade geográfica se espalha não só para os seus vizinhos imediatos, mas por todas as outras unidades.

Já no modelo com defasagem espacial, acrescenta-se entre as variáveis explicativas do modelo clássico (I) uma componente $\rho \mathbf{W}$ $\Delta \mathrm{y}_{\mathrm{i}}$, ou seja, a média dos valores da taxa de crescimento das unidades espaciais vizinhas. Assim: 
Modelo III - Convergência Absoluta com Defasagem Espacial

$$
\mathrm{f} \Delta \mathrm{y}_{\mathrm{i}}=\mathrm{x}_{\mathrm{i}}+\beta \mathrm{y}_{\mathrm{i}, 1991}+\rho \mathbf{W} \Delta \mathrm{y}_{\mathrm{i},+} \varepsilon_{\mathrm{i}}
$$

No caso presente, espera-se que $\rho>0$, sugerindo a existência de autocorrelação espacial positiva. A justificativa é que o crescimento da vizinhança de uma área tenda mais a contribuir para o seu crescimento econômico do que para deprimi-la.

Como se sabe, a $\beta$-convergência absoluta implica que todas as unidades sob análise estejam convergindo para o mesmo estado estacionário. Já na $\beta$-convergência condicional tais unidades convergem para diferentes estados estacionários, em função de características que lhes são específicas. Foram inseridas proxies, ${ }^{6}$ conforme apresentado em Pimentel e Haddad (2004), com o intuito de captar diferenças significativas das economias das unidades analisadas: uma para estoque de capital humano (média dos anos de estudo da população) e outra para urbanização (taxa de urbanização). Para o primeiro caso, baseamo-nos no fato de que níveis sensivelmente diferentes de qualidade de mão-de-obra tendem a atrair atividades igualmente diferentes no que se refere à utilização dos fatores de produção. Ademais, baseamo-nos no fato de que economias com diferentes níveis de urbanização terão capacidade diferenciada de oferecer (des)vantagens relativas à aglomeração econômica. Estimou-se, então, regressão expressa pela fórmula:

Modelo IV - Convergência Condicional Clássica

$$
\mathrm{d} \Delta \mathrm{y}=\mathrm{x}_{\mathrm{i}}+\beta \mathrm{y}_{1991}+\mathrm{Est}_{1991}+\mathrm{Urb}_{1991}+\varepsilon_{\mathrm{i}, \mathrm{t}}
$$

Onde:

Est $_{1991}=$ Log dos anos de estudo da população 1991

$\mathrm{Urb}_{1991}=$ Log da taxa de urbanização 1991

$$
\begin{aligned}
& \text { Modelo V - Convergência Condicional com Erro Espacial } \\
& \Delta \mathrm{y}=\mathrm{x}_{\mathrm{i}}+\beta \mathrm{y}_{1991}+\mathrm{Est}_{1991}+\mathrm{Urb}_{1991}+(\mathrm{I}-\lambda \mathrm{W})^{-1} \varepsilon_{\mathrm{i}}
\end{aligned}
$$

Modelo VI - Convergência Condicional com Defasagem Espacial

$$
\Delta \mathrm{y}=\mathrm{x}_{\mathrm{i}}+\beta \mathrm{y}_{1991}+\mathrm{Est}_{1991}+\mathrm{Urb}_{1991}+\rho \mathrm{W} \Delta \mathrm{y}+\varepsilon_{\mathrm{i}}
$$

\footnotetext{
6 Também foram estimados testes com densidade demográfica e taxa de analfabetismo, mas em nenhum dos testes a variável apresentou significância estatística.
} 
Não é incomum que os estimadores $\rho$ e $\lambda$ referentes aos modelos de defasagem e de erro sejam ambos estatisticamente significativos e que ambos os modelos sejam aceitos nos testes usuais. A questão que se põe é, portanto, como escolher qual é o modelo adequado. Para tanto, optou-se por seguir os padrões propostos por Florax, Folmer e Rey (2003). De acordo com esses autores, verificada a significância dos dois modelos, utiliza-se como critério para escolha o valor dos indicadores LIK (maximized log likelihood), AIC (Akaike infomation criteria) e SC (Schwartz criteria). A teoria indica que o modelo espacial mais adequado vai apresentar o maior valor para o indicador LIK e os menores valores para os indicadores AIC e SC.

\subsubsection{Resultados de convergência por municípios}

Primeiramente, testou-se a ocorrência de convergência absoluta sem qualquer atenção para os aspectos espaciais (Modelo I). Conforme sugere a teoria, o estimador $\beta$ foi negativo e significativo. Essa regressão, mesmo mal-especificada, é tomada como apenas como benchmark para as comparações com os modelos de convergência condicional e com relações espaciais que foram estimados a seguir. Também no sentido de apenas ter especificações como referência, os Modelos II e III testam a adequação do modelo de convergência absoluta com erros e defasagens espaciais, respectivamente. Nos dois casos, mostra-se que a hipótese de que os erros gerados na regressão são independentes é falsa e é necessário considerar as relações espaciais.

O Modelo IV, por sua vez, testa a existência de convergência condicional (ainda sem efeitos espaciais). Os resultados sugerem que os altos níveis de renda inicial dos municípios têm efeitos negativos sobre o crescimento. A variável que representa a qualificação do capital humano (Est1991) tem efeito positivo sobre a variação da renda per capita no período. A variável referente à urbanização (Urb1991) tem efeito negativo sobre a variação da renda per capita. Tal resultado indica a ocorrência do que chamamos de deseconomias de aglomeração. Salienta-se que tal resultado pode representar o sucesso econômico de municípios essencialmente rurais, com renda oriunda de atividades agropecuárias. Em relação à espe-cificação de convergência absoluta, nota-se o aumento sensível do valor do estimador $\beta$ e do $R^{2}$, conforme seria esperado. Todos esses resultados sugerem que, no Rio Grande do Sul no período 1991-2000, a hipótese de convergência absoluta é inadequada; o processo é de ocorrência de convergência condicional. Contudo, ainda se supõe que os erros da regressão sejam independentes. 
Os resultados de significância e sinal do Modelo de Convergência Condicional com Erro Espacial (Modelo V) repetem os do modelo IV: nível de renda com efeito negativo, indicando convergência; a média dos anos de estudo com efeito positivo, indicando que maior estoque de capital humano remete a um crescimento mais acelerado; e taxa de urbanização com efeito negativo, indicando a ocorrência de deseconomias de aglomeração. O componente de erro espacial também se mostrou significativo, demonstrando a falsidade da hipótese de que os erros gerados na regressão são independentes.

Finalmente, no modelo de convergência condicional com defasagem espacial (Modelo VI), os sinais das variáveis explicativas são os mesmos apresentados e explicados nos modelos anteriores. $\mathrm{O}$ componente de defasagem espacial mostrou-se significativo e positivo, indicando que o crescimento da renda dos municípios vizinhos eleva a velocidade do crescimento do município analisado.

Assim como já havia sido apresentado no modelo clássico, nas regressões que incluem os parâmetros espaciais, a hipótese de convergência absoluta se mostrou inadequada. Em todos os modelos, as proxies foram significativas, aumentaram a velocidade de convergência e o ajustamento do modelo, sugerindo que a hipótese de convergência condicional é a correta. Também ficou claro que o modelo clássico é inadequado, já que os dois testes espaciais mostraram-se significativos e apresentaram melhorias nos parâmetros das regressões. Resta agora especificar qual dos dois modelos espaciais é o mais adequado.

Seguindo o critério de escolha de especificações proposto, os indicadores LIK, AIC e SC sugerem que o mais apropriado o modelo espacial de autocorrelação dos erros. 
Tabela 1.Convergência Absoluta e Condicional com erros e Lag espaciais Nota: valores T (modelo clássico) e Z (modelos espaciais)entre parênteses.

\begin{tabular}{|c|c|c|c|}
\hline & MODELO I & MODELO II & MODELO III \\
\hline \multicolumn{4}{|c|}{ Convergência Absoluta } \\
\hline$\beta$ & $\begin{array}{l}-0,0294 \\
(-11,8954)\end{array}$ & $\begin{array}{l}-0,0341 \\
(-13,4243)\end{array}$ & $\begin{array}{l}-0,0274 \\
(-11,2788)\end{array}$ \\
\hline$\lambda$ & & $\begin{array}{l}0,4037 \\
(6,7226)\end{array}$ & \\
\hline$\rho$ & & & $\begin{array}{l}0,2585 \\
(4,3936)\end{array}$ \\
\hline $\mathrm{R}^{2}$ & 0,26 & 0,34 & 0,30 \\
\hline LIK & & 1241,08 & 1229,75 \\
\hline AIC & & $-2478,17$ & $-2453,49$ \\
\hline \multirow[t]{2}{*}{ SC } & & $-2469,88$ & $-2441,05$ \\
\hline & MODELO IV & MODELO V & MODELO VI \\
\hline \multicolumn{4}{|c|}{ Convergência Condicional } \\
\hline$\beta$ & $\begin{array}{l}-0,0432 \\
(-9,6707)\end{array}$ & $\begin{array}{l}-0,0587 \\
(-14,97786)\end{array}$ & $\begin{array}{l}-0,0414 \\
(-11,02805)\end{array}$ \\
\hline$\lambda$ & & $\begin{array}{l}0,4692 \\
(8,3018)\end{array}$ & \\
\hline$\rho$ & & & $\begin{array}{l}0,1721 \\
(2,9192)\end{array}$ \\
\hline Est $_{1991}$ & $\begin{array}{l}0,0578 \\
(7,3576)\end{array}$ & $\begin{array}{l}0,0668 \\
(8,683166)\end{array}$ & $\begin{array}{l}0,0527 \\
(7,305309)\end{array}$ \\
\hline Urb $_{1991}$ & $\begin{array}{l}-0,0058 \\
(-3,9779)\end{array}$ & $\begin{array}{l}-0,0028 \\
(-2,182463)\end{array}$ & $\begin{array}{l}-0,0048 \\
(-3,734161)\end{array}$ \\
\hline $\mathrm{R}^{2}$ & 0,36 & 0,44 & 0,37 \\
\hline LIK & & 1277,82 & 1256,53 \\
\hline $\mathrm{AIC}$ & & $-2547,66$ & $-2503,05$ \\
\hline SC & & $-2531,07$ & $-2482,32$ \\
\hline
\end{tabular}

Fonte: cálculos dos autores. 


\subsubsection{Resultados de Convergência por Conselhos Regionais de}

Desenvolvimento

De maneira similar aos testes com a base municipal, optou-se por testar primeiramente a ocorrência de convergência absoluta no modelo clássico (Modelo I'), desconsiderando o aspecto espacial. De acordo com o esperado, o estimador $\beta$ foi negativo e significativo. Sabe-se que tal modelo não é o apropriado, portanto partiuse para uma análise de convergência condicional, tanto no modelo clássico, quanto nos modelos espaciais. Com o intuito apenas de ter especificações como referência, os Modelos II' e III' testam a adequação do modelo de convergência absoluta com erros e defasagens espaciais, respectivamente.

Em relação à ocorrência de convergência condicional, o Modelo IV' nos mostra que a renda per capita inicial afetou negativamente o crescimento das unidades analisadas. Ademais, os COREDEs que apresentavam - no início do período - maior qualificação de capital humano e menor urbanização obtiveram crescimento mais acelerado que os demais. As três variáveis explicativas incluídas no modelo são estatisticamente significativas, como demonstra a tabela a seguir. Em relação à especificação de convergência absoluta, notase o aumento considerável do valor do estimador $\beta$ e do $\mathrm{R}^{2}$. Os resultados para COREDEs também sugerem que no Rio Grande do Sul no período 1991-2000 a hipótese de convergência absoluta é inadequada; há ocorrência de convergência condicional.

No que diz respeito aos modelos espaciais (Modelos V' e VI'), constata-se que os componentes adicionais (erro espacial e defasagem espacial) ao modelo de regressão clássica não são individualmente significativos, de acordo com o teste t. Tal ocorrência indica que, para o período analisado, os COREDEs gaúchos não têm seu crescimento espacialmente associado, ou seja, não há necessidade de se utilizar o instrumental da econometria espacial. 
Tabela 2. Convergência Absoluta e Condicional com erros e Lag espaciais Nota: valores T (modelo clássico) e Z (modelos espaciais)entre parênteses.

\begin{tabular}{|c|c|c|c|}
\hline & MODELO I' & MODELO II' & MODELO III' \\
\hline \multicolumn{4}{|c|}{ Convergência Absoluta } \\
\hline Constante & $\begin{array}{l}-0,0018 \\
(-0,9617)\end{array}$ & $\begin{array}{l}-0,0018 \\
(-0,5823)\end{array}$ & $\begin{array}{l}-0,0027 \\
(1,7640)\end{array}$ \\
\hline$\beta$ & $\begin{array}{l}-0,0229 \\
(-4,1221)\end{array}$ & $\begin{array}{l}-0,0197 \\
(-4,1793)\end{array}$ & $\begin{array}{l}-0,0199 \\
(-4,1660)\end{array}$ \\
\hline$\lambda$ & & $\begin{array}{l}0,5812 \\
(2,8968)\end{array}$ & \\
\hline$\rho$ & & & $\begin{array}{l}0,4885 \\
(2,7389)\end{array}$ \\
\hline $\mathrm{R}^{2}$ & 0,45 & 0,60 & 0,60 \\
\hline LIK & 80,28 & 82,60 & 82,95 \\
\hline AIC & $-156,56$ & $-161,21$ & $-159,91$ \\
\hline \multirow[t]{2}{*}{ SC } & $-154,38$ & $-159,03$ & $-156,64$ \\
\hline & MODELO IV' & MODELO V' & MODELO VI' \\
\hline \multicolumn{4}{|c|}{ Convergência Condicional } \\
\hline Constante & $\begin{array}{l}-0,0476 \\
(-1,9313)\end{array}$ & $\begin{array}{l}-0,0519 \\
(-2,2423)\end{array}$ & $\begin{array}{l}-0,0394 \\
(-1,6596)\end{array}$ \\
\hline$\beta$ & $\begin{array}{l}-4,15738 \mathrm{e}-005 \\
(-1,1242)\end{array}$ & $\begin{array}{l}-6,57543 e-005 \\
(-1,7600)\end{array}$ & $\begin{array}{l}-4,02997 \mathrm{e}-005 \\
(-1,1885)\end{array}$ \\
\hline$\lambda$ & & $\begin{array}{l}0,3355 \\
(1,2696)\end{array}$ & \\
\hline$\rho$ & & & $\begin{array}{l}0,2080 \\
(0.9695)\end{array}$ \\
\hline Est $_{1991}$ & $\begin{array}{l}0,0294 \\
(1,6937)\end{array}$ & $\begin{array}{l}0,0364 \\
(2,1232)\end{array}$ & $\begin{array}{l}0,0247 \\
(1,4642)\end{array}$ \\
\hline Urb $_{1991}$ & $\begin{array}{l}-0,0298 \\
(-4,4062)\end{array}$ & $\begin{array}{l}-0,0260 \\
(-4,1829)\end{array}$ & $\begin{array}{l}-0,0262 \\
(-4,0784)\end{array}$ \\
\hline $\mathrm{R}^{2}$ & 0,69 & 0,71 & 0,71 \\
\hline LIK & 86,44 & 86,77 & 86,95 \\
\hline $\mathrm{AIC}$ & $-164,89$ & $-165,53$ & $-163,91$ \\
\hline SC & $-160,52$ & $-161,17$ & $-158,45$ \\
\hline
\end{tabular}

Fonte: cálculos dos autores. 


\section{Análise de Kernel Estocástico para COREDEs e Municípios Gaúchos}

A partir da crítica de Friedman (1992) e das contribuições de Quah (1993a, 1993b, 1996) disseminou-se a utilização de métodos de kernel estocástico no exame da dinâmica do crescimento econômico. Com isso pode-se ter uma outra visão das alterações da distribuição durante o período 1991-2000. Seguindo os passos da literatura sobre o tema, foi calculada o logaritmo da renda relativa per capita municipal e utilizou-se um kernel gaussiano com bandwidth baseado na regra de Silverman (1986). ${ }^{7}$

A figura 7 contém os resultados da análise referentes aos municípios gaúchos entre 1991 e 2000. O formato alongado das curvas de nível evidencia que os municípios gaúchos tenderam a preservar suas posições na distribuição.
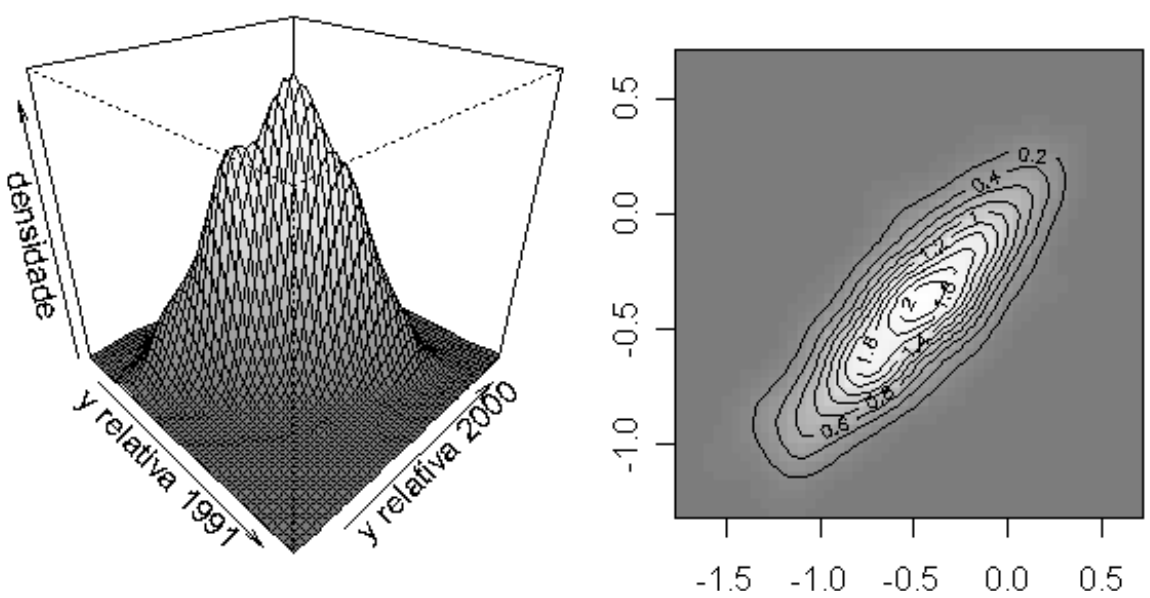

Figura 7. Kernel Estocástico e Curva de Nível - Municípios (1991-2000) Fonte: Cálculos dos autores.

Já a figura 8 representa os resultados da aplicação de kernel estocástico para os coredes no mesmo período. Como se vê, o perfil da função de densidade probabilidade é bastante distinto apresentando elevações nos dois extremos (ricos-ricos e pobres-pobres) que inexiste quando os dados são examinados ao nível municipal.

\footnotetext{
7 Foi utilizado o software R 2.3.1 e seus diversos pacotes auxiliares
} 

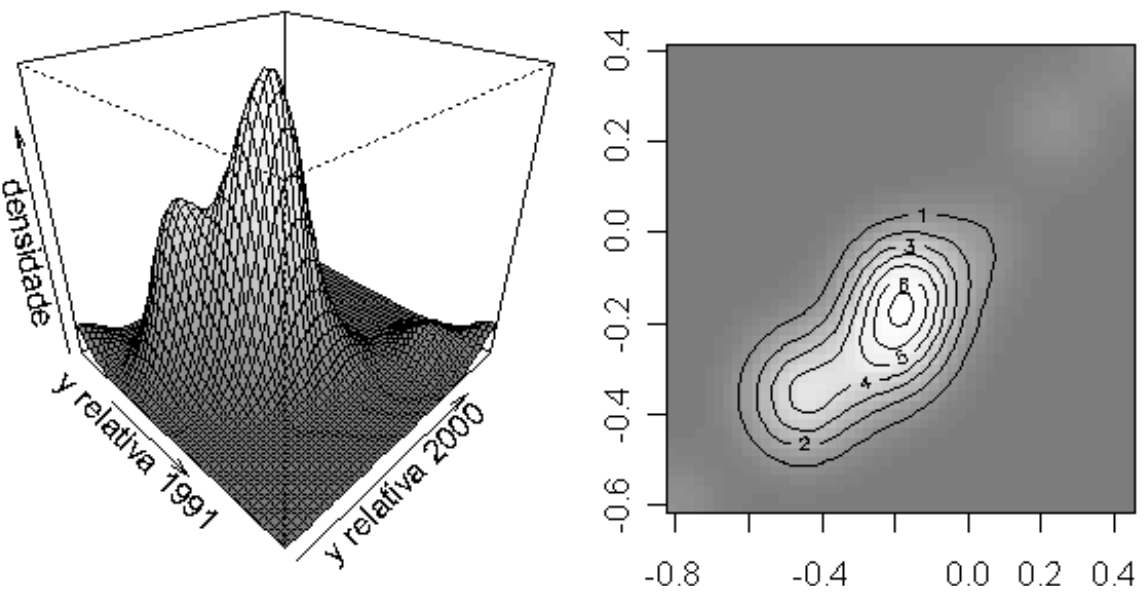

Figura 8. Kernel Estocástico e Curva de Nível - Coredes (1991-2000) Fonte: Cálculos dos autores.

\section{Considerações Finais}

Este trabalho buscou aplicar o instrumental da análise e econometria espacial à questão da distribuição pelo território gaúcho da renda per capita, com destaque para o problema da unidade de área modificável, o MAUP.

A lei de Tobler sustenta que "everything is related to everything else, but nearby things are more related than distant things" (TOBLER, 1970, p. 236). Bem de acordo com essa Lei, o trabalho mostrou que os indicadores de associação espacial e a necessidade da econometria espacial são mais intensos quando se analisam os municípios do que os COREDEs. Os testes econométricos de ß-convergência com base na malha municipal estão mal-especificados se não levarem em conta a autocorrelação espacial. No caso presente, o modelo mais adequado de correção foi o de erro-espacial e não o de lag-espacial. Já aplicação de kernel estocástico, mostrando que o formato das funções de densidade de probabilidade dos COREDEs e municípios gaúchos foram razoavelmente distintas, reforçou a importância de atentar para o MAUP.

Uma recomendação geral é, sempre que viável, trabalhar com diversos níveis de análise espacial e comparar os resultados. Manter ao longo de uma pesquisa uma única delimitação territorial do objeto pode ser bastante enganoso. Devido ao MAUP, as conclusões sempre são condicionadas ao nível de análise espacial esco- 
lhido. A realidade não é como um fractal que repete o mesmo padrão desde as macro-regiões até as áreas censitárias. O pesquisador, além de respeitar as restrições de ordem prática, deve ser sensato ao limitar o tipo e o número de níveis geográficos a examinar. No caso presente, como se viu, clusters de rendas baixa e alta teriam desaparecido se os COREDEs fossem o único nível examinado. ${ }^{8}$

Além disso, quer na análise dos municípios, quer na dos COREDES, mostrou-se a necessidade de observar os dados com mais atenção. Como se está em uma economia com livre mobilidade de trabalhadores, fenômenos não observados nos estudos internacionais de convergência podem ocorrer. Mostrou-se aqui que unidades que perderam população apareceram como partes de clusters de alto crescimento da renda per capita e como convergindo em relação aos mais ricos. Ora, uma elevação da renda per capita por redução das capitas e não por um aumento do estoque de capital que um mecanismo de convergência à la Solow sugeriria. Note-se que a crítica é muito mais do que apontar a ocorrência da Falácia de Galton (não obstante, esta também ter sua participação), mas destacar que os dados indicam mecanismos que não se coadunam com a previsão da teoria.

Se, por um lado, o programa de pesquisa de convergência está exausto, por outro, ainda falta muito para compreender os processos espaciais da dinâmica regional. Neste sentido, os recursos de análise exploratória de dados espaciais, dos quais aqui se teve apenas uma amostra, agem na direção de fornecer um retrato mais fiel de tal dinâmica. A utilização dessas ferramentas pelos economistas proporciona não só novos recursos de visualização, mas também novos testes e indicadores de dinâmica regional que pressupõem a utilização de bases de dados georreferenciadas, como os propostos por Rey e Janikas (2004). Este novo conjunto permitirá - esperase - que os economistas regionais considerem cada vez mais os aspectos espaciais em suas análises empíricas e não apenas em suas teorias.

\footnotetext{
\& A técnica de point-pattern analysis é imune ao MAUP (O'SULLIVAN e UNWIN, 2003). O procedimento considera as distâncias entre os eventos e dispensa qualquer tipo de agregação espacial. Infelizmente, a base de dados aqui utilizada não é adequada para sua utilização. Ver Duranton e Overman (2002) para uma aplicação econômica de point-pattern analysis.
} 


\section{Bibliografia}

ALONSO, J. A. F. . Evolução das desigualdades inter-regionais de renda interna no Rio Grande do Sul. 1939-70. 2. ed. Porto Alegre: FEE, 1986. 161 p.

ALONSO; AMARAL, R.Q. Desigualdades Intermunicipais de Renda no Rio Grande do Sul: 1985-2001.

ALONSO, J. A. F. ; BENETTI, M. D. ; BANDEIRA, P. S. . Crescimento econômico da região sul no Rio Grande do Sul: causas e perspectivas. $1^{\mathrm{a}}$. ed. Porto Alegre, RS: Fundação de Economia e Estatística Siegfried Emanuel Heuser, 1994. 229 p.

ANSELIN, L. Spatial econometrics: Methods and models. Boston: Kluwer Academic, 1988. 93-115, 1995

Local Indicators of Spatial Association — LISA, Geographical Analysis 27:

Spacestat tutorial: A workbook for using Spacestat in the analysis of spatial data. Urbana Illinois, 1992.

BAGOLIN, I. P., GABE, J. E RIBEIRO, E. P. Crescimento e Desigualdade no Rio Grande do Sul: uma revisão da Curva de Kuznets para os municípios gaúchos (1970-1991). Anais do XXX Encontro Nacional de Economia - ANPEC, Nova Friburgo, dezembro, 2002.

BANDEIRA, P. S. As raízes históricas do declínio da região sul. In: ALONSO, J. A. F.;BENETTI, M. D.; BANDEIRA, P. S. Crescimento econômico da região sul do Rio Grande do Sul: causas e perspectivas. Porto Alegre: FEE, 1994.

BARRO, R.; SALA-I-MARTIN, X. Economic Growth. New York: McGraw-Hill, 1995.

BAUMOL, W. J. Productivity growth, convergence, and welfare: What the long-run data show. American Economic Review, v. 76, n. 5, p. 1072-85, 1986.

BÊRNI, D. Á.; MARQUETTI, A.; KLOECKNER, R. A desigualdade econômica do Rio Grande do Sul: primeiras investigações sobre a curva de Kuznets. Anais do $1^{\circ}$. Encontro de Economia Gaúcha - PPGE-PUCRS e FEE, Porto Alegre, Maio, 2002.

CARLUER, F.; GAULIER, G. Archipelago Europe: Geographic and structural analysis of convergence. In: Innovation and Growth: new challenges for the regions 2002, Sophia-Antipolis. 2002.

DURANTON, G.; OVERMAN, H. Testing for Localization Using Micro-Geographic Data, Center for Economic Policy Research Discussion Paper \# 3379. 2002.

FLORAX, R. J. G. M.; FOLMER, H.; REY, S. J. Specification searches in spatial econometrics: The relevance of Hendry's methodology. Regional Science and Urban Economics. September, v. 33, n. 5, p. 557-79, 2003.

FRIEDMAN M. Do old fallacies ever die? Journal of Economic Literature, 30, 1992, p. 2129-132.

FUENTE, A. de la. Notas sobre la economia del crescimiento. Barcelona: Universitat Autònoma, 1996.

HAINIING, Robert. Spatial Data Analysis: theory and practice. London: Cambridge University, 2003. 
MAGALHÃES, A. Clubes de convergência no Brasil: Uma abordagem com correção espacial. In: Anais do XXIX Encontro Nacional de Economia 2001, Salvador. 2001.

MAGALHÃES, A.; HEWINGS, G.; AZZONI, C. Spatial dependence and regional convergence in Brazil. Working Paper REAL 00-T-11, Urbana, 2000.

MARQUETTI, A. A.; RIBEIRO, E. P. Determinantes do desempenho econômico dos municípios do Rio Grande do Sul, 1991-2000. Secretaria de Coordenação e Planejamento do Rio Grande do Sul, 2002.

MONASTERIO, LM e ÁVILA, R. P. Economic Growth in Southern Brazil: Rio Grande do Sul (1939-2001). Workshop on Spatial Econometrics (Kiel, Germany). Disponível em: http://www.uni-kiel.de/ifw/konfer/spatial/prel-program.htm. 2005

MOSSI, M. et al. Growth dynamics and space in Brazil. European Regional Science Association Conference Papers. Disponível em: http://www.ersa.org/ersaconfs/ersa02/ cd-rom/papers/499.pdf. 2002.

O'SULLIVAN D, D. J. UNWIN. Geographic Information Analysis Wiley: Hoboken, NJ. 2003.

PIMENTEL, E. A.; HADDAD, E.A Desigualdades regionais em Minas Gerais: análises espaciais do fenômeno, 1991-2000. Trabalho apresentado no III Encontro da Associação Brasileira de Estudos Regionais. Belo Horizonte, Junho de 2004.

PORTO, JÚNIOR, S. S. Dinâmica de crescimento e convergência de renda per capita no Brasil: o caso da região Sul. Tese de doutorado não publicada. Universidade Federal do Rio Grande do Sul, 2000.

PÔRTO JÚNIOR, S. S. ; RIBEIRO, Eduardo Pontual . Dinâmica de crescimento Regional - uma análise empírica para a região Sul. Revista Econômica do Nordeste, Fortaleza-CE, v. 31, p. 454-483, 2000.

QUAH, D. Spatial Cluster Empirics. Working Paper incompleto. Disponível em: http://econ.lse.ac.uk/staff/dquah/p/200306sce-2pp.pdf, 2003.

Galton s Fallacy and Tests of the Convergence Hypothesis, The Scandinavian Journal of Economics, 95, 1993a, p. 427-443.

. Empirical cross-section dynamics in economic growth, European Economic Review, 37, 1993b, p. 426-434. 40, 951-958, 1996

RAMIREZ, M. T.; LOMBOGUERRERO, A. M. Spatial dependence and economic growth: Evidence from a panel of countries. 2002.

REY, S. J.; MONTOURI, B. D. U.S. Regional income convergence: A spatial econometric perspective. Regional Studies, v. 33, p. 143-156, 1999.

. Spatial Dependence in the Evolution of Regional Income Distributions.

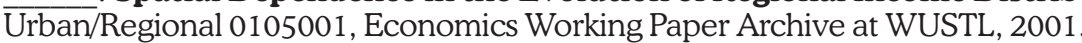

SILVERMAN B. W. Density estimation for statistics and data analysis, Monographson Statistics and Applied Probability 26, Chapman and Hall, London. 1986. 
SOUZA, N. J. . Estrutura Espacial das Atividades Econômicas do Rio Grande do Sul, 1990/2000. In: II ENCONTRO DE ECONOMIA GAÚCHA, 2004.

TOBLER, W. R. A computer model simulation of urban growth in the Detroit region. Economic Geography, v. 46, p. 234-240, 1970.

VERNER, D. E TEBALDI, E. Convergence, Dynamics, and Geography of Economic Growth: The Case of Municipalities in Rio Grande do Norte, Brazil. World Bank Working Paper, n. 3302. Disponível no site: http://econ.worldbank.org/view. php?type $=5$ Ëid $=35586$. May, 2004. 
